\title{
Formalizing Manufacturing Process Modeling using Composition Trees
}

\author{
Lian Wen ${ }^{1, a}$, David Tuffley ${ }^{1, b}$ \\ ${ }^{1}$ School of ICT, Nathan Campus, Griffith University, Brisbane, Qld, Australia \\ I.wen@griffith.edu.au, ${ }^{\mathrm{b}}$ d.tuffley@griffith.edu.au
}

Keywords: manufacturing process, manufacturing process modeling, composition trees, behavior engineering, process optimization.

\begin{abstract}
Optimizing manufacturing process modeling is critical to improving the efficiency and quality of manufacturing. However, manufacturing processes in general can be very complicated. A manufacturing process may involve (a) transforming a material into a new form, (b) combining different materials to form a new object, and (c) retrieving a certain part from a material as a new entity. All those activities could be confusing because many intermediate objects that exist only for a short period during the manufacturing process may have no formal names. To solve this problem, this paper proposes that composition trees (CT) can be successfully used to model manufacturing processes. Composition trees are parts of the formal notation in behavior engineering (BE), which is an innovative approach to the development of large software systems. For a composition tree, its characteristics of being intuitive, expressive and formal give it many advantages over other formal and informal modeling languages in software engineering. This paper uses a cooking case study to demonstrate how to use a composition tree model manufacturing processes. It is argued that the method could be applied to process engineering to good effect.
\end{abstract}

\section{Introduction}

Manufacturing processes generally consist of many activities. Raw materials pass through various operations and are eventually transferred into finished products [14]. Manufacturing processes cover many different industrial domains, e.g. the classic handbook for mechanical engineering [7] introduces a large number of manufacturing processes including sand casting, molding, rolling, shearing bending, welding, boring, drilling etc., for different materials such as plastic, metals and wood. Modern manufacturing processes are becoming complex and increasingly characterized by high levels of automation, integration and various human supervisory controls [10]. This trend demands the model of manufacturing process to be formalized.

Formalizing manufacturing process modeling has several advantages leading to greater efficiency, including: (a) a process will be easy to understand and verify [21]; (b) it can be simulated [3], analyzed, processed and optimized [14] by automation tools [11], and (c) eventually increasing the process flexibility [9], efficiency and quality of manufacturing.

There exist several modeling techniques such as GRAI [12], IDEFF0 [2], SADT [13], SSADM [17] related to manufacturing process modeling. However, most of the modeling techniques are based on traditional flow chart style modeling languages used in the tool of Arena [11].

Flow charts, firstly introduced into industrial engineering in 1920s [8], have been a popular software process modeling tool for more than half century and not without good reason. However, its general graphic structure and the lack of the concept of component and object make it difficult to effectively model large and complex software intensive systems. Behavior Engineering (BE) [1], as a new software engineering approach, introduces two modeling languages called behavior trees (BT) [4] and composition trees (CT) [5].

In the last decade, vigorous research shows that behavior trees and composition trees are powerful modeling languages; they can be used to solve a broad range of software engineering problems including requirement defect detection [6], software change and evolution [18], software process modeling, process verification [19] and many more, which can be found at the BE website [1]. The 
reason that $\mathrm{BT}$ and $\mathrm{CT}$ are so powerful in solving different complex problems is largely due to the characteristics of BT and CT being intuitive, so problems modeled by BT and CT can be easily understood and verified. It is expressive, so it can model different types of problem. It is formal, so there is no ambiguity in the model and which lends itself to being processed by software tools.

Given the advanced features of BT and CT, it is not unreasonable to expect that this innovative technique can be useful for other domains including that of manufacturing process modeling.

In this paper, we use a cooking process case study to demonstrate that composition trees can be used to model manufacturing processes and handle the proper naming problem.

A manufacturing process could produce some intermediate objects that appear for a short period in the process. Many of those intermediate objects may not have a formal name [20]; therefore, the description of the process in natural languages can be ambiguous and confusing. For example, in a cooking process, a grapefruit is juiced, then should it be called the juice or the grapefruit? When the juice and some sugar is mixed, what should we call the new material?

The proper name problem is not new. It can be traced far back as the classical Greek period. The philosopher Plutarch proposed the paradox of the Ship of Theseus which sought an answer to the question how much of something needs to be replaced before it is no longer the original thing? The Ship of Theseus was preserved over time by replacing components as they wore out or decayed, even down to the individual planks from which the ship was built. After a time, no part of the original ship remained. The question was unresolved. Some philosophers argued that the ship remained the same, and others contended that it was not the same [15].

Traditionally, these kinds of problems are handled casually when defining a manufacturing process. The lack of formalism inevitably hinders the efficiency and quality of the process especially when the manufacturing processes are becoming highly complex and need to be simulated and optimized by software tools.

In this paper, we use a case study to demonstrate how a composition tree modeling can solve three different types of problems, which are common in manufacturing processes: (a) one material is transformed into a new form and may have a new name, (b) different materials are combined to form a new object, which may or may not have an official name, and (c) something is retrieved from an existing material as a new entity.

\section{Case Study}

This section presents a case study to show how to model a manufacturing process in a composition tree. The manufacturing process is a simplified receipt to make a cold dish called spring fruit salad. The original recipe can be found in the Internet [16]. Because the syntax and semantics of a composition tree is very intuitive, we will not formally introduce them in this paper, apart from using the case study to explain them in an informal way. The detailed syntax and semantics of composition trees can be found elsewhere [19]. The receipt is shown in Table 1 and the corresponding composition tree is shown in Figure 1.

Table 1. The receipt of spring fruit salad

\begin{tabular}{|l|l|}
\hline Ingredient (IG) & Procedures \\
\hline - 1 large pink grapefruit & S1. Juice the grapefruit. Add the juice into the sugar. \\
- 1 cup white sugar & S2. Heat sugar until sugar becomes syrup. \\
- 1 vanilla bean & S3. Cut vanilla bean and scrape seeds out. Add seeds to syrup. \\
- $1.3 \mathrm{~kg}$ seedless watermelon & S4. Remove skin from watermelon. Cut flesh into 2cm cubes. \\
& S5. Drizzle (cubes) with syrup. \\
\hline
\end{tabular}

Figure 1 is the Composition Tree of the process to make spring fruit salad. The root node (at the top of the diagram) is the final product (it is also called system in software engineering). Directly under the root nodes, there are five nodes representing five materials (called components in software engineering) for the final product. 


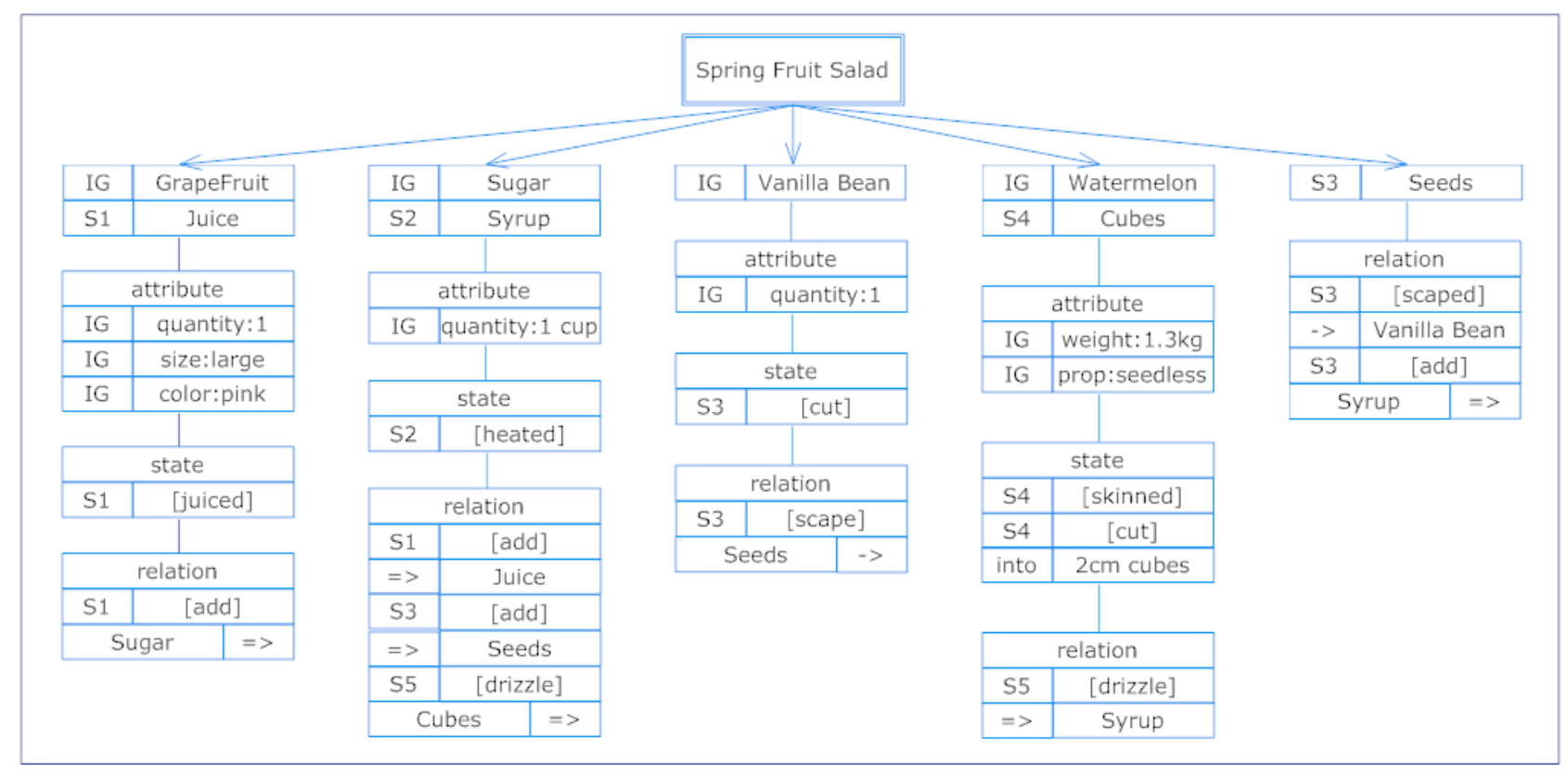

Figure 1. The Composition Tree of Spring Fruit Salad

We start from the leftmost branch of the tree. The top node includes two rows. The first row has two cells; the right cell "GrapeFruit" is the name of the material; the left cell "IG" means this name firstly appears in the ingredient section in the receipt (see Table 1). The second line includes two cells, "S1" and "Juice", that mean the material is also called Juice from S1 (Step1). The node under the name node shows the attributes of the material, which is large and pink. Similarly this information is retrieved from the "IG" part in the receipt. Then the next node shows all the states of the material described in the process. It says in "S1", the grapefruit will be in the state of "juiced". The last node shows the relations of this material with others materials. The meaning of the node is that, in "S1", the grapefruit (also called juice) is added into sugar. The symbol of " $=>$ " on the right side means this material is mixed into other materials and the original material will not have an identity anymore.

The second branch describes the material of Sugar, also called Syrup from S2. The attribute node and state node are very simple, so we only explain the relation node. The relation node shows that in S1, Juice is added into Sugar; in S3, Seeds are added into Sugar. The symbol of "=>" on the left side means some other materials are added into the material. Finally, in S5, the material of Sugar (it is also called syrup with juice and seeds in it) is drizzle (mixed) into Cubes.

The third branch is for Vanilla Bean. The only special node is the relation node. The symbol "->" on the right side means some part of the material has been removed to create or added into another material. The difference between " $=>$ " and " $->$ " is that for " $=>$ ", the identity of the original material disappear, but "->" will not destroy the identity of the original material. Therefore, after the seeds are scraped from the vanilla bean, the bean still exists.

There is no new syntax in the fourth branch. In the last branch, one interesting point is that the material Seeds are firstly introduced in S3 not in IG; also it is created from a vanilla bean.

\section{Discussion and Conclusion}

The previous section uses a simple cooking process to demonstrate how to use a composition tree to model a manufacturing process. Through this case study, we can see that all the three common problems, which are (a) one material is transformed into a new form and may have a new name, (b) different materials are combined to form a new object, and (c) something is retrieved from an existing material as a new entity. All three have been solved in a simple and natural way.

This positive result suggests that a composition tree could be a promising formal language to model manufacturing processes. Of course, a composition tree only shows the statistic aspect such as 
attribute, state and relation of individual materials. We may need behavior trees [18] to model the dynamical aspects of manufacturing processes and that will be one of the future research topics.

\section{References}

[1] Behavior Engineering Web Site, http://www.behaviorengineering.org/ [2011 July]

[2] A. Cheng-Leong, K.L. Pheng, and G. Leng, "IDEF*: A comprehensive modelling methodology for the development of manufacturing enterprise systems", Int'1 Jounral of Production Research (v37), pp 3839-3858, 1999

[3] D. Curcio, F. Longo, G. Mirabelli, Manufacturing Process Management Using a Flexible Modeling and Simulation Approach, WSC'07 Proceedings of the $39^{\text {th }}$ conference on Winter simulation, 2007

[4] Dromey, R.G., "From Requirements to Design : Formalising the Key Steps", (Invited Keynote Address), IEEE International Conference on Software Engineering and Formal Methods, SEFM'2003

[5] Dromey, R. G., System Composition: Constructive Support for the Analysis and Design of Large Systems, SETE-2005, Systems Engineering/Test and Evaluation Conference, Brisbane, Australia, 2005

[6] Dromey, R.G, Powell, D., “Early Requirements Defects Detection”, TickIT International, 4Q05, 2005, pp. 3-13

[7] C. Fennell, R. Shivpuri, et al. Mark's Standard Handbook for Mechanical Engineers, $10^{\text {th }}$ ed, Chapter 13 Manufacturing process, ISBN-10: 0070049971, McGraw-Hill Professional, 1996

[8] Flow chart, http://en.wikipedia.org/wiki/Flowchart [July, 2011]

[9] K. Hallenborg, A. J. Jensen, Y. Demazeau: Reactive agent mechanisms for Manufacturing process control, IEEE/WIC/ACM, pp399-403 2007

[10] S. H. Huang, J. P. Dismukes et al: Manufacturing system modelling for productivity improvement, Jounral of Manufacturing Systems, V21, Issue 4, pp 249-259

[11] W.D. Kelton, R.P. Sadowski, D.A. Sadowski: Simulation with Arena, New York: WCB McGrawHill, 1998

[12] I. McCarthy, M. Menicou, A Classification schema of manufacturing decision for the GRAI Enterprise Modelling, Computers in Industry, Vol 47, 3, pp 339-355, 2002

[13] K. Santarek, I.M. Buseif, Modelling and design of flexible manufacturing systems using SADT and Petri nets tools, Journal of Manterials Processing Techology (v76), pp212-218, 1998

[14] V. Sarhangian, H.Eskandari, M. Ardakani: Optimizing inspection strategies for multi-stage manufacturing processes using simulation optimization, Proceedings of the 2008 Winter Simulation Conference, pp 1974-1980, 2008

[15] Ship of Theseus http://en.wikipedia.org/wiki/Ship_of_Theseus [July, 2011]

[16] Spring Fruit Salad, http://www.taste.com.au/recipes/7962/spring+fruit+salad [July, 2011]

[17] K.T.K Toh, Modelling architectures: A review of their application in structured methods of information system specification, Int'l Jounral of Production Research (v37) pp 1439-1458, 1999

[18] Wen, L., Dromey, R.G., "From Requirements Change to Design Change: A Formal Path", Proceedings of the 2nd IEEE International Conference on Software Engineering and Formal Methods, pp. 104-113, 2004

[19]L. Wen, D. Tuffley, T. Rout, Using Composition Trees to Model and Compare Software Process, in Proceedings of $11^{\text {th }}$ International Conference, SPICE2011, pp1-15, 2011

[20] Y. Yamakata, T. Funatomi, et al. in: A Study of Object Naming According to the Manufacturing processes in Cooking Activity, CEA'09 Proceedings of the ACM multimedia 2009 workshop on cooking and eating activities (2009)

[21] G. Zülch, Modelling and Simulation of Human Decision-Making in Manufacturing System, Proceedings of the Winter Simulation Conference, pp 947-953, 2006 